\title{
Histomorphometry and Stereological Study of Septomarginal Trabecula in Pig's Heart
}

\author{
Sergio Ricardo Rios Nascimento,, ${ }^{1,2}$ Cristiane Regina Ruiz, ${ }^{2}$ Marina Nunes, ${ }^{1}$ Samara Urban de Oliva'
}

'Department of Morphology and Genetics - Developmental Biology Laboratory - Federal University of São Paulo (UNIFESP), São Paulo, SP, Brazil ${ }^{2}$ Human's Anatomy Department, São Camilo University Center, São Paulo, Brazil

Disclose and conflicts of interest: none to be declared by all authors

\begin{abstract}
Introduction: cardiovascular disease is one of the main causes of morbidity and mortality worldwide. Important knowledge about the cardiovascular system was acquired through the use of experimental research and requires choosing a suitable model. The similarities in anatomy and physiology between pigs and humans, swine have emerged as a model for preclinical protocols. The septomarginal trabecula (ST) is an extremely important fibromuscular structure located in the right ventricle of most species. Variations in the ST anatomical and histological structures has a significant cardiac physiology and clinical importance. The purpose of this study was to perform morphometry and stereology analyzes of the ST in right ventricles of pig's hearts.
\end{abstract}

Material and Methods: fourteen pig hearts were analyzed macroscopically to obtain an anatomical description and length and thickness measurements. For histological and stereological analysis were also performed.

Results: the swine hearts presented the ST as a tubular fibromuscular band with significant variation in length and diameter. Variations were also observed in the number of fixations (single, bifurcated, quadrifurcated). The volume density of the tissues present in the ST showed the predominance of cardiac muscle tissue followed by connective tissue. The stereological analyzes showed also significant differences in the amount of cardiac muscle and connective tissue in thin and thick structures. Conductive fibers were surrounded by the connective tissue and, were more peripherally located in the thick ST compared to the thin ST.

Conclusion: in pig hearts, morphological and histological variations found in ST suggest possible correlations with changes in cardiac physiology.

Keywords: Heart; Pig; Septomarginal trabecula; Stereology.

\section{Introduction}

Cardiovascular disease is one of the main causes of morbidity and mortality worldwide. Normal anatomical and histological features should be known for the diagnosis and development of medical treatments for cardiovascular anomalies. Important knowledge about the cardiovascular system was acquired through the use of experimental research. However, extrapolating data from animals to humans requires choosing an experimental model with similar morphology and physiology. The similarities in size, coronary anatomy, and physiology between pigs and humans, swine have emerged as a model for preclinical protocols ${ }^{1-5}$.

Meantime, detailed information on interindividual and interspecies variations related to cardiac anatomy and histology of the various structures is still lacking, both in humans and in the most common large mammalian animal models, and these variations may interfere with cardiovascular function ${ }^{6-8}$.

The septomarginal trabecula (ST), also known as moderator band, is an extremely important fibromuscular structure which connects the interventricular septum and the right ventricular anterior wall, or at the base of the anterior papillary, of most species including humans, dogs, pigs, bovines, goats, sheep and equines. Its function is to prevent distension of the right ventricle and to transmit the right branch of the atrioventricular bundle to the right ventricle. Furthermore, the ST has an important role in the contraction and relaxation of the ventricles ${ }^{8-22}$.

Several anatomical studies reported that an anomalous ST has important clinical repercussions involving the right ventricle and atrial and ventricular septum. Thus, some probable pathologies occurring within the band, most often of ischaemic aetiology, can constitute an important aetiology of conduction disturbances of the heart ${ }^{23}$.

In addition to presenting differences in origin and insertion, the number of insertions and shapes of the ST can differ between species. Although structural composition of the ST structure has a significant cardiac physiology and clinical importance, few papers precisely describe its histological composition ${ }^{24}$. Although some publications present the ST histological characterization, there is no detailed analysis of the quantification and distribution of the tissues which constitute the ST.

Considering the importance of using animals as a model in studies and in the development of 
techniques with applications in the clinical practice and heart surgery in humans, a more detailed macro and microanatomic knowledge of the heart of these animals is necessary, mainly regarding the cardiac muscle and conductive fibers of the ST, which would be involved in electrophysiological processes of the hearts. Thus, the purpose of this study was to perform morphometry and stereology analyzes of the ST in right ventricles of pig's hearts.

\section{Material and Methods}

A total of fourteen pig hearts were assessed. The experimental protocol followed the ethical principles adopted by the Brazilian College of Animal Experimentation and was approved by the local Institutional Ethics Committee (CEUA/UNIFESP Protocol number 8942220816).

Each piece was numbered and photographed. An incision was performed in the inferior border of the right auricle, at the base of the pulmonary trunk origin, along the right margin of the heart to the apex of the right ventricle, avoiding to cut the ST in its marginal fixation. Each ST was macroscopically examined for anatomical description of its form and fixations on the right margin and the interventricular septum; then, length and diameter were carefully measured with a Mitutoyo $^{\circledR}$ digital caliper (150 mm Absolute Digimatic model).

The ST were then classified for diameter and length according to the values of the interquartile intervals obtained, respectively, as follows: $\leq \mathrm{IQ1} \mathrm{mm}=$ Thin / short; between IQ1 and IQ3 = Medium / median; $\geq$ IQ3 $\mathrm{mm}=$ Thick / long.

Fragments from the middle third of each ST were collected and fixed by immersion in Bouin's liquid for 24 hours and prepared for Paraplast Plus-embedded (P-3683, Sigma Chemical Co., St. Louis, MO). Five micrometers thick cross sections were obtained and stained with Masson Trichrome and submitted to the Periodic Acid-Schiff histochemical method with Harris's Hematoxylin counterstaining (PAS+H).

The ST transversal sections were analyzed using a light microscope attached to the Leica Qwin-V3 (Leica,
Cambridge, UK) image analysis system $\times 20$ objective lens. The histological composition and proportions of connective and muscle tissues and the conduction myofibrils were observed by descriptive analysis.

Analyses of volume density (Vv) of the connective tissue, cardiac muscle fibers, vascular tissues and conduction myofibrils were performed using the Leica QWin image analysis system. Twenty-two fields were systematically and randomly sampled, and the images were captured using a digital camera connected to a light microscope. The Principle of Delesse states that the planimetric fraction of a section occupied by sections of a given component corresponds to the fraction of the tissue volume occupied by this component ${ }^{25}$. For that reason, according to the basic law of stereology, the density of area of the profiles $\left(A_{A}\right)$, which measures the relative occupation of testarea for the area of the images of the structure under evaluation, is comparable to the $\mathrm{Vv}$, i.e, the density of profiles in relation to the space. Consequently, Vv and $\mathrm{A}_{A}$ have similar interpretations ${ }^{25-27}$. Thus, from captured images of the connective tissue, cardiac muscle fibers, vascular tissues and conduction myofibrils, the areas were delineated. Each specific Vv was respectively obtained by the ratio of the delineated area to the total area of the ST tissue analyzed ${ }^{28}$.

The data was subjected to the non-parametric Wilcoxon test for one sample, using GraphPad Instat ${ }^{\circledR}$ software. The Kruskal-Wallis test, followed by the Dunn's test, was used to compare Vv of the tissue components of the ST different diameters. The differences were considered statistically significant when $p \leq 0.05$.

\section{Results}

The swine hearts evaluated presented the ST as a tubular fibromuscular band with variable length and diameter; these differences were statistically significant (Table 1). The ST classified as thin showed long or medium lengths, but none was considered short; ST with medium diameter usually had medium lengths. However, all the ST with thick diameter presented short lengths (Table 2).

Table 1. Total length and diameter (in $\mathrm{mm}$ ) of ST from pigs' hearts.

\begin{tabular}{c|c|c}
\hline & Length $(\mathbf{m m})$ & Diameter $(\mathbf{m m})$ \\
\hline Median (IQ1-IQ3) & $21.26(16.36-32.53)^{\mathrm{a}}$ & $3.56(2.16-5.58)^{\mathrm{a}}$ \\
\hline Minimum & 15.36 & 1.02 \\
\hline Maximum & 35.32 & 7.56 \\
\hline
\end{tabular}

${ }^{a} p \leq 0.05 ; n=14$.

Table 2. Correlation between length and diameter measurements of porcine ST.

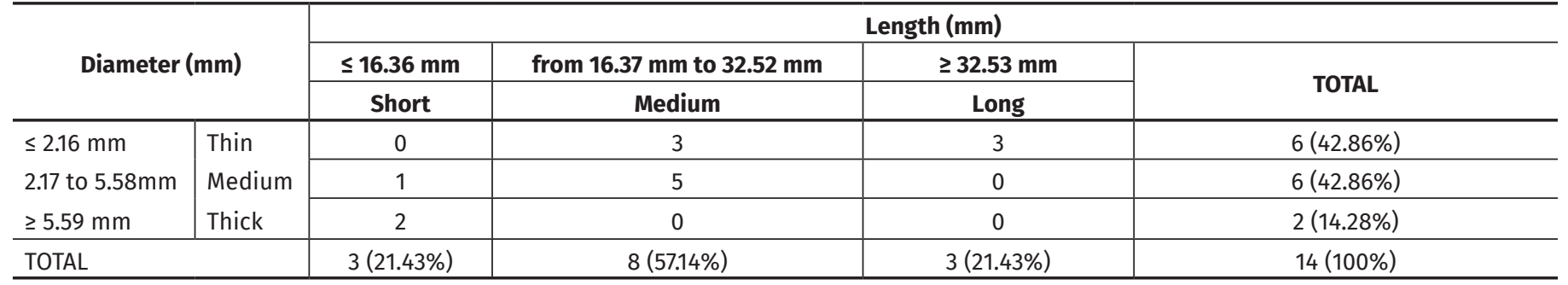


The septal insertion was in the interventricular septum (IV) and the marginal insertion in the right ventricular margin (RV) topography, at the base or near the papillary muscle. The trajectory performed by the structure between insertion points showed various patterns: rectilinear, curved and arcuate. Anatomical variations were also observed in the number of fixations. Although the majority of the samples presented a single ST (78,57\%), two specimens presented a ST with two marginal fixations (bifurcated) and, in one sample, the ST had a septal fixation and four marginal fixations (quadrifurcated) (Figures 1a and 1b, respectively).
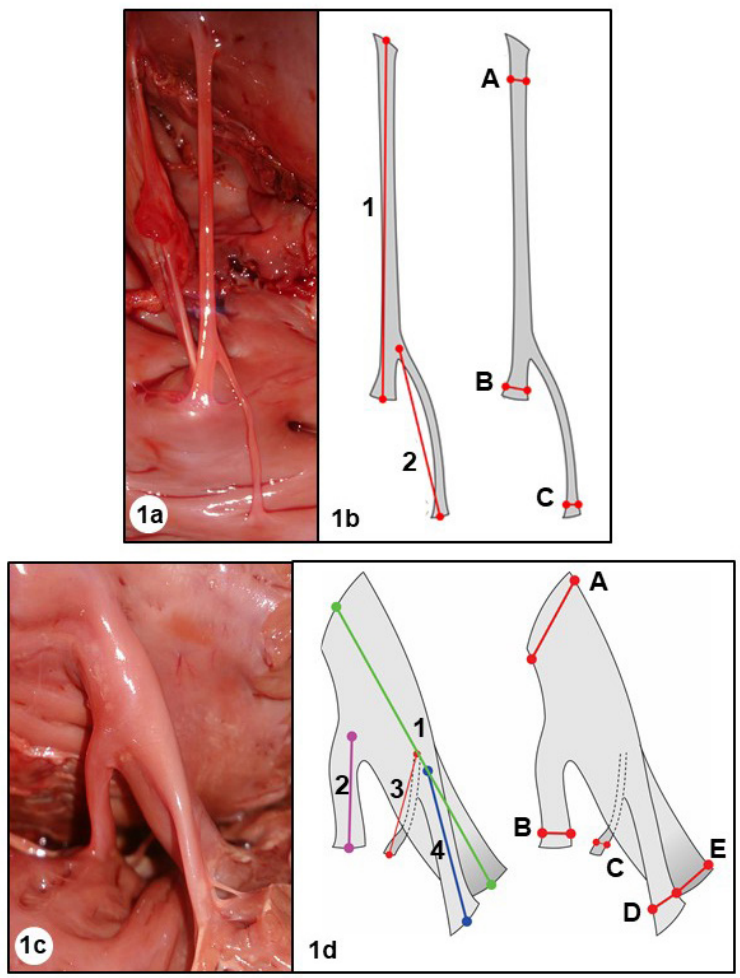

Figure 1. Illustrative diagram of the swine ST; a and b) bifurcated, with septal fixation ( $A$ ) and two marginal fixations (B and $C)$. Length $1(A-B): 33.44 \mathrm{~mm}$; length 2: $18.20 \mathrm{~mm}$. Diameter A: $1.91 \mathrm{~mm} ; \mathrm{B}: 1.36 \mathrm{~mm}$; $: 0.82 \mathrm{~mm}$. C and d) quadrifurcated, with septal fixation (A) and four marginal fixations (B, C, D and E). Length 1: 32.23 mm; length 2: 16.25 mm; length 3: 13.01; length 4: 12.65. Diameter A: $5.51 \mathrm{~mm}$; B: $1.93 \mathrm{~mm}$; C: $0.75 \mathrm{~mm}$; D:2.19 mm; E: $5.51 \mathrm{~mm}$.

Histological analysis showed that the ST consisted of cardiac muscle fibers, connective tissue, vascular tissue and conduction myofibrils (Purkinje fibers). Differences in the distribution of ST muscle fibers and connective tissue were observed when comparing samples of smaller thickness, which presented connective tissue predominance and cardiac muscle tissue sparse (Figures $2 \mathrm{a}$ and $2 \mathrm{~b}$ ), and the largest thickness samples, which presented a predominance of cardiac muscle tissue (Figures $2 \mathrm{c}$ and $2 \mathrm{~d}$ ). Conductive fibers were surrounded by the connective tissue and, were more peripherally located in the thick ST compared to the thin ST (Figures $2 \mathrm{~b}$ and 2d).

Similarly, in ST with more than one marginal fixation, there were histological differences between the branches of the same trabeculae; thicker branches presented predominance of cardiac muscle, while the thinner branches were mainly constituted by connective tissue (Figure 3).
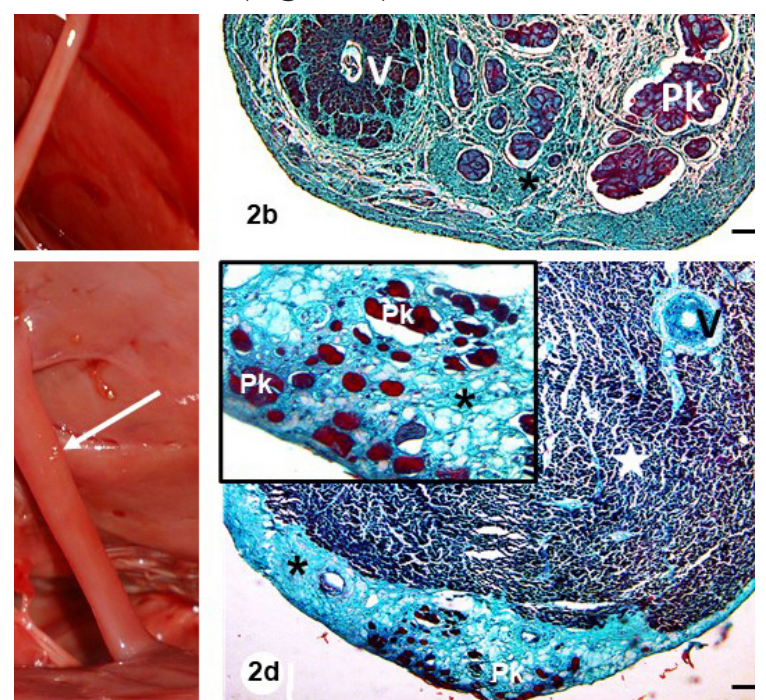

Figure 2. a) Digital image of a thin ST (arrow). b) Photomicrograph of transversal section of the ST, showing a large amount of connective tissue (asterisks). c) Digital image of a thick ST (arrow). d) Photomicrograph of the cross-sectional ST demonstrating a wide distribution of cardiac muscle fibers (star) and a reduced amount of connective tissue (asterisks). Inset: In detail, the occurrence of peripherical Purkinje cells surrounded by connective tissue. V: blood vessel; Pk: Purkinje fibers. Paraplast. Masson trichrome. Bars: $450 \mu \mathrm{m}$ (figure 2b), 160 $\mu \mathrm{m}$ (figure 2d) and $64 \mu \mathrm{m}$ (inset figure $2 \mathrm{~d}$ ).
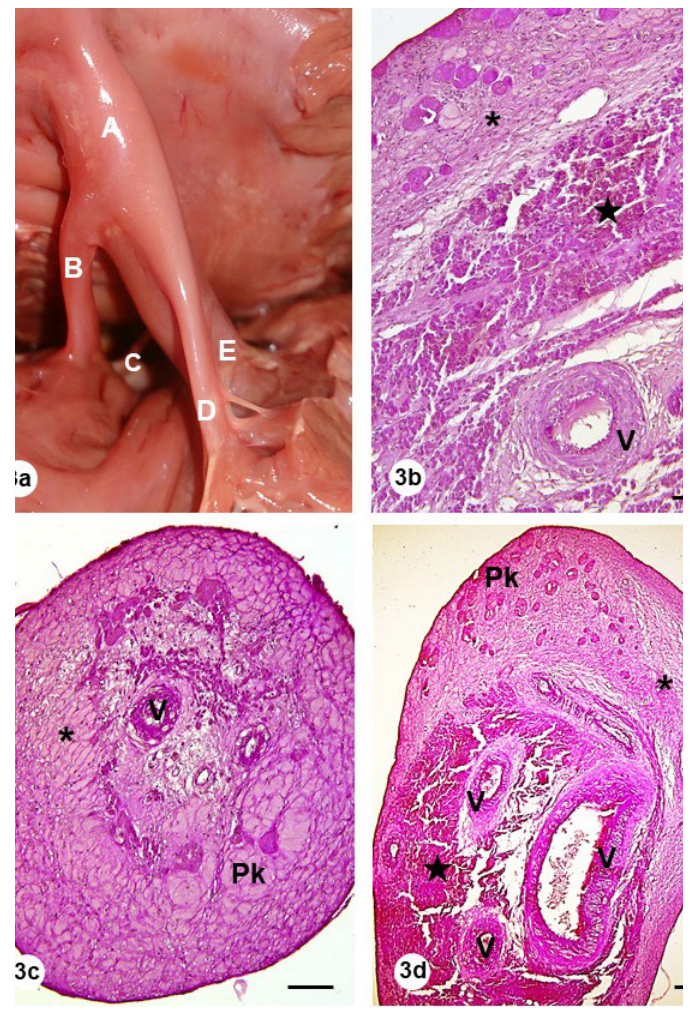

Figure 3. a) Digital image of a quadrifurcated ST. b) Photomicrograph of a crosssectioned part of the thick branch of the quadrifurcated swine heart ST (E branch indicated in figure 3a). Note the presence of cardiac muscle fibers (star), Purkinje fibers ( $\mathrm{Pk}$ ) peripherally arranged and surrounded by connective tissue (asterisk). $\mathrm{V}$ : blood vessel. c) Photomicrograph of a cross-sectioned part of the thin branch of the quadrifurcated ST (B branch indicated in figure 3a) showing a large proportion of connective tissue (asterisk) and Purkinje fibers (Pk), blood vessels (V) and scarce cardiac muscle tissue. d) Sectioned photomicrograph of the thin branch of the ST (C branch indicated in figure $3 \mathrm{a}$ ) showing vascularization branches (V), the presence of Purkinje cells (Pk) located between the collagen fibers (asterisk) and cardiac muscle fibers (star). Paraplast. PAS. Bars: $30 \mu \mathrm{m}$, $75 \mu \mathrm{m}$ and $88 \mu \mathrm{m}$, respectively. 
The Vv of the tissues present in the ST showed the predominance of cardiac muscle tissue followed by connective tissue (Figure 4A). However, according to the histological analysis of trabeculae with different diameters, the stereological analyzes showed significant differences in the amount of cardiac muscle and connective tissue in thin and thick structures. Thus, higher Vv of connective tissue and few cardiac muscle tissue was presented in the thin trabeculae, while the thicker samples presented higher $\mathrm{Vv}$ of cardiac muscle tissue (Figure 4B).

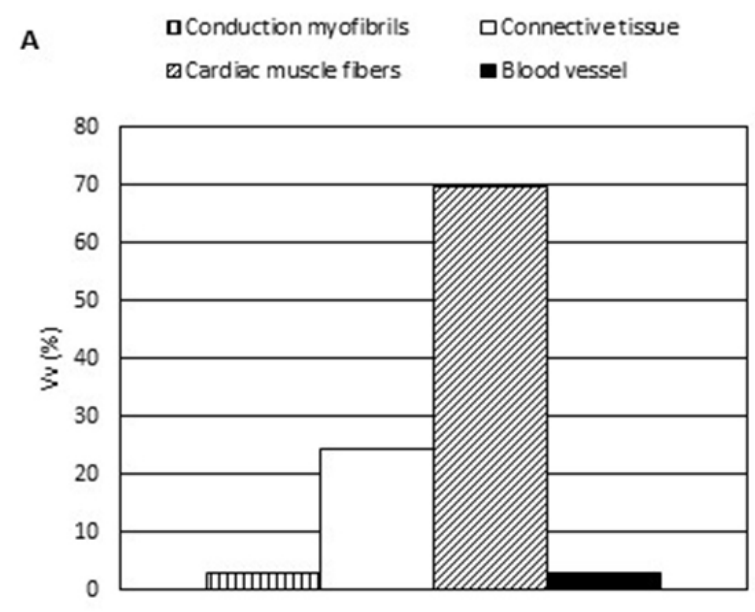

and shapes, and several morphological classifications of the moderator band (MB) have been proposed ${ }^{6-8,12}$, $15,38,40$. These variations show that attention should be given to the ST topography, since this structure is a crucial part of the conducting system and can therefore have major clinical consequences when it comes to congenital abnormalities.

In this study, the length and thickness of the pig ST also varied. Correlation between these parameters was noted, and larger trabeculae had thinner diameters; In contrast, thicker diameter ST were generally

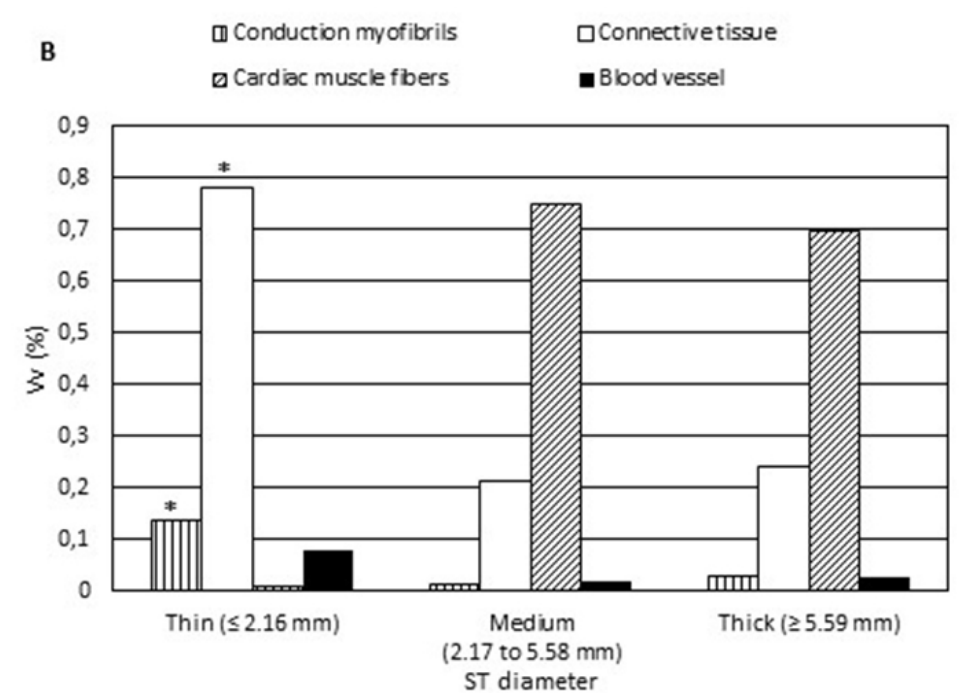

Figure 4. A) Vv of the cardiac muscle fibers, connective tissue, blood vessels and Purkinje fiber of pigs ST. B) Comparison between Vv of the cardiac muscle fiber, connective tissue, vascular tissue and Purkinje fiber (conduction myofibril) between porcine ST of thin, medium and thick diameters. Values expressed as percentage. Statistically significant differences: * $\mathrm{p} \leq 0.05$.

\section{Discussion}

Understanding the heart's morphophysiology and cardiovascular diseases through knowledge of cardiac anatomy, histology and physiology in both humans and animal models is essential. The pig has been widely used as a model in cardiovascular research. However, knowledge about the comparative cardiac morphology of pigs is limited ${ }^{29}$.

Structurally, the right ventricle in the pig is very similar to the human except for the $\mathrm{ST}^{30}$. In human and animal heart ventricles, ST primarily provides quick transmission of electric impulses to the parietal wall and prevents excessive dilatation of the ventricles during diastole ${ }^{31,32}$. Clinical significance of the ST is reported to be associated with conduction disturbances of the heart ${ }^{12,33-38}$, so much so that ST removal has been performed for the treatment of idiopathic premature ventricular contractions ${ }^{35,39}$. Besides, iatrogenic heart disease has been related to ST excision, evidencing the importance of careful handling during surgical procedures ${ }^{6,23,39}$.

This study showed histological and anatomical intraspecies variation on the pig ST, including insertion's number, shape, length, as well as the diameter and how the tissue constitution may differ between samples. Previous studies also observed variations on ST lengths shorter. Several other studies demonstrated variable morphological and topographical patterns of ST in different species ${ }^{16,17,23,41}$. Loukas et al..$^{6}$ observed highly varied patterns in porcine ST, and classified them according to their surface and area measurements into categories: type I, short and coarse; type II, long and thick; type III, short and thin; type IV, long and thin.

Regarding the number of fixations, the results of this study showed that most of the swine ST were unique. However, in two samples, variations in the number of fixations occurred only at the right ventricularrelated extremity and presented bifurcated and quadrifurcated. This finding has also been reported by other researchers in pigs ${ }^{17}$, horses ${ }^{19}, \operatorname{dog}^{16,42}$ and even humans ${ }^{12,15,18}$.

In this study, the histological examination of the ST revealed the presence of connective tissue, cardiac muscle fibers, blood vessels, and Purkinje cells in pig heart samples, in agreement with the histological description of the trabeculae in other animal species and in humans $\mathbf{s}^{11,16,17,19,32,43-46}$. It was also evidenced a variation in Purkinje fibers distribution and disposition, besides the proportion between the striated cardiac and connective muscle tissues between the samples. Bojsen-Moller and Tranum-Jensen ${ }^{9}$ described that the ST of pigs varies widely in cardiac muscle quantity, which were absent in 5 of 19 trabeculae examined. It was 
shown that in the presence of cardiac muscle fibers, Purkinje fibers were located only in the periphery; On the other hand, when the ST did not present muscular fibers, the Purkinje fibers were located in the central part of the $\mathrm{ST}^{9}$, demonstrating that the muscle bundle and the stimulus complex branch are dissociated in the ST of pigs ${ }^{14}$. Bombonato et al. ${ }^{19}$ also related that the structural arrangement of the ST in deer, baboons and foxes is composed solely of conduction tissue and absence of cardiac muscle, suggesting the occurrence of several varied histological characteristics between different species.

Preparations of isolated MB from pigs have shown that the muscular compartment is sufficiently well coupled and organized to allow electrical propagation ${ }^{47}$. In sheep, Walton et al. ${ }^{37}$ demonstrated that the ST structure is composed of two excitable and uncoupled compartments: Purkinje fibers and myocardium. The Purkinje fibers were separated from the myocardium in a coaxial configuration by lipid deposits and extensive extracellular collagen surrounding individual bundles. The Purkinje fiber/ muscle ratios were highly variable and may be explained by a high intraspecies variability of the $M B$ thicknesses.

\section{Conclusion}

It is suggested that, in pig hearts, ST varying diameters differ in their histologic constitution: in the thinner trabeculae, there is a predominance of connective tissue and in the thicker trabeculae, there is a predominance of cardiac muscle tissue. However, there seems to be no alteration in the amount of Purkinje fibers. Thus, morphological and histological variations found in ST suggest possible correlations with changes in cardiac physiology.

\section{Acknowledgements}

The authors thank CAPES (Coordenação de Aperfeiçoamento de Pessoal de Nível Superior) for financial support.

\section{References}

1. Hearse DJ, Sutherland FJ. Experimental models for the study of cardiovascular function and disease. Pharmacol Res 2000;41(6):597-603

2. Cui J, Li J, Mathison M, et al. A clinically relevant large-animal model for evaluation of tissue-engineered cardiac surgical patch materials. Cardiovasc Revasc Med 2005;6(3):113-120

3. Milani-Nejad N, Janssen PM. Small and large animal models in cardiac contraction research: advantages and disadvantages. Pharmacol Ther 2014;141(3):235-249

4. Camacho P, Fan H, Liu Z, He JQ. Large Mammalian Animal Models of Heart Disease. J Cardiovasc Dev Dis 2016;3(4):30

5. Oh JG, Ishikawa K. Experimental Models of Cardiovascular Diseases: Overview. Methods Mol Biol 2018;1816:3-14

6. Loukas M, Klaassen Z, Tubbs RS, et al. Anatomical observations of the moderator band. Clin Anat. 2010;23(4):443-450

7. Raghavendra AY, Kavitha, Arunachalam K, Pratik T, Harsha CR. Anatomical study of the moderator band. NUJHS 2013;3(4):78-81

8. Shenoy $P$, Lucas M, Vinay KV, Ramos A. Morphometric study of septomarginal trabecula (moderator band). Int J Anat Res 2016; 4(4):3302-3308

9. Bojsen-Moller F, Tranum-Jensen J. On nerves and nerve endings in the conducting system of the moderator band (septomarginal trabecula). J Anat 1971;108(Pt 3):387-395

10. Ansari A, Ho SY, Anderson RH. Distribution of the Purkinje fibres in the sheep heart. Anat Rec 1999;254(1):92-97

11. Sathyamoorthy OR, Ramesh G. Microanatomical studies on the moderator band (trabecula septomarginalis) of horses (Equus caballus). J Vet Anim Sci 2008; 39: 33-35

12. Kosiński A, Koztowski D, Nowinski J, et al.. Morphogenetic aspects of the septomarginal trabeculae in the human heart. Arch Med Sci 2010;6(5):733-743

13. Leão CR, Pacha DL, Cyriaco T, et al. Anatomy of the septomarginal trabecula in goat hearts. IJAE. 2010; 115(3): 229-234

14. Rocha H, Elizario LFE, Wafae GC, Silva NC, Ruiz CR, Wafae N. Anatomy of the septomarginal trabécula in Landrace pig hearts. Morphologie 2010;94(305):26-29

15. Bandeira STF, Wafae GC, Ruiz C, Nascimento SR, Fernandes JR, Wafae N. Morphological classification of the septomarginal trabécula in humans. Folia Morphol 2011;70(4): 300-304

16. Bombonato PP, Mariana ANB, Borelli V, et al. Morphometric study of trabecula septomarginalis in dogs. Ars Veterinaria

\section{2;28(4):250-254}

17. Gulyaeva AS, Roshchevskaya IM. Morphology of moderator bands (septomarginal trabecula) in porcine heart ventricles. Anat Histol Embryol 2012;41(5):326-332

18. Mamatha H, Shenoy D, Souza ASD, Prasanna LC, Sumalatha S. A morphometric study on the septomarginal trabeculaee in South indian cadavers. RRJMHS 2013;2(2): 65-70

19. Bombonato PP, Agreste FR, Nogueira K, dos Santos AC, Viana ARDCB, de Lima EMM. Stereometry Study of Septomarginal Trabeculae in Equines. J Vet Adv 2015;5(4):874-878

20. Paraskevas G, Koutsouflianiotis K, Iliou K. The first descriptions of various anatomical structures and embryological remnants of the heart: A systematic overview. Int J Cardiol 2017;227:674-690 21. Chagas C, Pires L, Leite T, Babinski M. The artery of Mouchet: blood supply of the septomarginal trabecula in 50 human hearts. Rev Assoc Med Bras (1992)2018;64(10):916-921

22. Nascimento SRR, Ruiz CR, de Oliva SU. Histological and anatomical variations of septomarginal trabecula in bovine hearts. Anat Histol Embryol 2019;48(3):228-233

23. Zajączkowski M, Kosiński A, Grzybiak M, et al. The structure of the vascular system of the septomarginal trabecula in the heart of an adult. Adv Clin Exp Med 2018;27(5):623-631

24. Haroun HS. Comparative Anatomy of the Cardiac Septomarginal Trabecula (Moderator Band). Anatomy Physiol Biochem Int J 2017;2(4), 555595

25. Weibel ER, Kistler GS, Scherle WF. Practical stereological methods for morphometric cytology. J Cell Biol 1966;30(1):23-38

26. Gundersen HJ, Bendtsen TF, Korbo L, et al. Some new, simple and efficient stereological methods and their use in pathological research and diagnosis. APMIS 1988;96(5):379-394

27. Mandarim-de-Lacerda CA. Stereological tools in biomedical research. An Acad Bras Cienc 2003;75(4):469-486

28. de Oliva SU, Miraglia SM. Carbamazepine damage to rat spermatogenesis in different sexual developmental phases. Int J Androl 2009;32(5):563-574

29. Crick SJ, Sheppard MN, Ho SY, Gebstein L, Anderson RH. Anatomy of the pig heart: comparisons with normal human cardiac structure. J Anat 1998;193(Pt 1):105-119

30. Morita T, Shimada T, Kitamura H, Nakamura M. Demonstration of connective tissue sheaths surrounding working myocardial cells and Purkinje cells of the sheep moderator band. Arch Histol Cytol 
1991;54(5):539-550

31. Abouezzeddine 0 , Suleiman M, Buescher T, et al. Relevance of endocavitary structures in ablation procedures for ventricular tachycardia. J Cardiovasc Electrophysiol 2010;21(3):245-254

32. Ateş S, Karakurum E, Takcı L, Başak F, Kürtül i. Morphology of the atrioventricular valves and related intraventricular structures in the wild pig (Sus scrofa). Folia Morphol (Warsz) 2017;76(4):650659

33. Jongbloed MR, Wijffels MC, Schalij MJ, et al. Development of the right ventricular inflow tract and moderator band: a possible morphological and functional explanation for Mahaim tachycardia. Circ Res 2005;96(7):776-783

34. Anter E, Buxton AE, Silverstein JR, Josephson ME. Idiopathic ventricular fibrillation originating from the moderator band. J Cardiovasc Electrophysiol 2013;24(1):97-100

35. Sadek MM, Benhayon D, Sureddi R, et al. Idiopathic ventricular arrhythmias originating from the moderator band: Electrocardiographic characteristics and treatment by catheter ablation. Heart Rhythm 2015;12(1):67-75

36. Li JY, Jiang JB, He Y, Luo JC, Zhong GQ. Ventricular Tachycardia Originating from Moderator Band: New Perspective on Catheter Ablation. Case Rep Cardiol 2017;2017:3414360

37. Walton RD, Pashaei A, Martinez ME, et al. Compartmentalized Structure of the Moderator Band Provides a Unique Substrate for Macroreentrant Ventricular Tachycardia. Circ Arrhythm Electrophysiol 2018;11(8):e005913

38. Lee JY, Hur MS. Morphological classification of the moderator band and its relationship with the anterior papillary muscle. Anat Cell Biol 2019;52(1):38-42

39. Kurosawa $\mathrm{H}$, Becker AE. Surgical anatomy of the atrioventricular conduction bundle in anomalous muscle bundle of the right ventricle with subarterial ventricular septal defect. Pediatr Cardiol 1985;6(3):157-160
40. Kosiński A, Nowiński J, Kozłowski D, Piwko G, Kuta W, Grzybiak $M$. The crista supraventricularis in the human heart and its role in the morphogenesis of the septomarginal trabecula. Ann Anat 2007;189(5):447-456

41. Cope LA. Morphological Variations in the Canine (Canis familiaris) Right Ventricle Trabecula Septomarginalis Dextra and a Proposed Classification Scheme. Anat Histol Embryol 2015;45(6):437-442

42. Armiger LC, Urthaler F, James TN. Morphological changes in the right ventricular septomarginal trabecula (false tendon) during maturation and ageing in the dog heart. J Anat 1979;129(Pt 4):805817

43. Kosiński A, Zajaczkowski M, Kuta W, Koztowski D, Szpinda M, Grzybiak M. Septomarginal trabécula and anterior papillary muscle in primate hearts: developmental issues. Folia Morphol 2013;72(3):202-209

44. Ghonimi W, Abuel-Atta AA, Bareedy $\mathrm{MH}$, Balah A. Gross and microanatomical studies on the moderator bands (septomarginal trabeculae) in the heart of mature Dromedary camel (Camelus dromedaries). J Adv Vet Anim Res 2014;1(2):24-31

45. Ghonimi WAM, Elbaz A, Ibrahim L, Khair NSB, Emam H, Hellal A. Moderator bands (Trabecula septomarginalis) of mature buffalo (Bos bubalis L.) with special emphasis on the structure and distribution of the Purkinje. Cell Dev Biol 2015;4(3):1-10

46. Emam MA, Abo-Ahmed Al. Age-related histomorphometric and immunohistochemical changes of the moderator band in Egyptian Baladi cattle. Anat Histol Embryol 2019;48(2):149-156

47. Gettes LS, Morehouse N, Surawicz B. Effect of premature depolarization on the duration of action potentials in Purkinje and ventricular fibers of the moderator band of the pig heart. Role of proximity and the duration of the preceding action potential. Circ Res 1972;30(1):55-66
Received: June 25, 2021

Accepted: August 8, 2021
Corresponding author Samara Urban de Oliva oliva.samara@unifesp.br; samaraurban@gmail.com 